\title{
Paired-associate performance on successive recall-test trials as a function of number of successive pairing trials and stimulus elements'
}

\author{
JOHN K. BERRY AND WILLIAM F, BATTIG \\ UNIVERSITY OF MARYLAND
}

Paired-associate (PA) performance uas significantly im proved by interpolation of three successive recall-test trials between pairing trials, but did not change systematically over the three successive test trials. Increasing number of stimulus elements had only slight delcterious effects on initial PA performance. The results indicate that the principal effect produced by interpolated test trials is to facilitate PA learning during subsequent pairing trials

One of the many disadvantages of the classical paired-associate (PA) anticipation procedure has been its inseparability of presentation of stimulus (S) terms for attempted response (R) anticipation from that of S-R pairs for learning (Battig, 1965). Through the temporal separation of these two functions that characterizes the recall method, the present experiment was designed to evaluate separately the cffects of the pairing and recall-test portions of the trial on PA learning and performance. By systematically varying the number of successive pairing trials preceding at set of three successive test trials, the existence and magnitude of any improvements in performance across successive test trials (Richardson \& Gropper, 1964) was also evaluated. In addition, the design included systematic variations in number of letters constituting the S-terms, as well as conditions involving prior free-recall learning of the S-terms, in order to determine whether increased stimulus learning may be the primary determinant of any changes in performance across successive test trials.

Method

The 110 Ss were introductory psychology students fulfilling a course requirement through their participation, who were assigned to one of 11 groups of $10 \mathrm{Ss}$ each according to a predetermined unsystematic sequence based on order of appearance in the laboratory, which provided that each group be represented once in each block of $11 \mathrm{Ss}$. Nine of the groups represented a complete factorial design with three numbers of successive pairing trials (one, three, or six, designated respectively as $R 1, R 3$, and $R 6$ conditions) and three numbers of elements constituting each PA S-term (one, two, or three, designated as $\mathrm{S} 1, \mathrm{~S} 2$, and $\mathrm{S} 3$ conditions). The remaining two $\mathrm{S} 3-\mathrm{R} 1-\mathrm{P}$ and $\mathrm{S} 3-\mathrm{R} 6-\mathrm{P}$ groups corresponded respectively to the $\mathrm{S} 3-\mathrm{R} 1$ and $\mathrm{S} 3-\mathrm{R} 6$ conditions, but with prior free-recall pretraining on each of the three-letter S-terms.
All Sis learned an eight-pair PA list with maximally similar but highly familiar words (BAG, BAT, BUG, BUT, RAG, RAT, RUG, RUT) as R-terms. The eight S3 S-terms were constituted from 24 different letters (cxcluding $A$ and $U$ ), and were equated both in Anderson (1965) association value for individual letters and in frequency of interletter associations (Underwood \& schulz, 1960). The $\$ 2$ and $\$ 1$ s-terms were produced by deleting letters from each $\$ 3 \mathrm{~s}$-term so as to maintain comparable letter and interletter association values. In an effort to maximize stimulus learning and performance differences under $\mathrm{S} 1, \mathrm{~S} 2$, and $\mathrm{S} 3$ conditions, each $\$ 2$ bigram was presented in both possible letter orders on alternate trials, and each $\$ 3$ trigram was presented equally often in each of three different letter orders which provided that each letter appear once in each of the three letter positions.

All lists were learned to the same criterion of errorless performance on the first two of the three successive test trials which followed every one, three, or six successive pairing trials under $R 1, R 3$, and $R 6$ conditions, respectively. All materials were presented at a 4-sec. rate by Carousel automatic slide projector with a 4-sec. interval between successive pairing trials and an 8- or 12-sec. interval between all other presentation series. A different unsystematic serial order was used each time through the list, and the s2 and s3 letter orders during all three test trials remained the same as for the immediately preceding pairing trial.

Pretraining for the S3-R1-P and S3-R6-P groups involved the successive 4-sec. presentation of all eight $\mathrm{S}$-terms, followed by $45 \mathrm{sec}$. for attempted oral recall by spelling with no restriction either on order in which the S-terms were recalled or on letter order. All three \$3 letter orders were presented equally often during $s$-term pretraining, which continued to a criterion of three correct recalls of each $\mathrm{s}$-term (which then was removed from the list).

Results

Number of errors summed over the three test trials following the sixth pairing trial decreased significantly $(p<.001)$ from $R 6(9.6)$ to $R 3(5.8)$ and $R 1$ (1.4) conditions, but neither the slight increase from S1 $(4.4)$ to S2 (6.1) and $S 3(6.4)$ conditions nor the interaction approached significance (both Fs $<1$ ). The R1 and R3 conditions did show a significant $(p<.05)$ difference after the third pairing trial between $S 1(6.8), S 2(8.5)$, and 
S3 (12.2) conditions, but by the fifth trial the S3-R1 group actually became slightly superior to S2-R1 and S1-R1 conditions.

Significantly $(p<.05)$ fewer errors were also made on the first three sets of test trials by the S3-R1-P (21.6) than the S3-R1 (36.0) group, and after six pairing trials by the S3-R6-P (4.1) than the S3-R6 (10.2) group. The two $\mathbf{P}$ groups also recalled significantly $(\mathrm{p}<.001)$ more stimulus letters than the S3-R1 and S3-R6 groups on a free written-recall test immediately following the last PA trial.

In none of the present conditions were there any systematic changes in performance across the three successive test trials. Over the first such set, where previous studies had typically obtained increases (Richardson \& Gropper, 1964), almost half (50) of the present Ss showed no change whatever from the first to the third successive test trial, whereas slightly but insignificantly more Ss declined (37) than improved (23), and for no group did this change approach significance (all Fs $<1$ ).

\section{Discussion}

While the present results are completely negative with respect to any improvement or other systematic change in performance across three successive recall-test trials, the marked decrement from R1 to $R 6$ conditions clearly demonstrates that interpolated test trials serve to facilitate performance following one or more subsequent pairing trials. Thus interpolated test trials appear to increase the amount learned during subsequent pairing trials, most likely by enabling $S$ to identify those pairs which he has not yet learned, and to concentrate upon these the next time(s) they are presented.

The present results contrast sharply with the several recent studies allegedly. demonstrating "learning" during recall-test trials (Richardson \& Gropper, 1964). Most of these studies, however, employed either the PA anticipation procedure or a lengthy retention interval preceding the successive test trials, both of which would be expected to produce extensive temporary dismuption on the first test trial with subsequent opportunity for "warm-up" improvement (Lazar \& Van Laer, 1966). Although not emphasized by the latter authors, they too found no significant changes over 10 successive test trials given immediately after 10 recall-method learning trials, as did Reynolds (1965) over five successive test trials after nine trials under both prompting and anticipation methods. Thus it is by no means clear that "using the alternate study and recall method, some changes in performance will occur even though the correct responses are not shown" (Underwood, 1966, p. 549). In the present study, such changes did not occur even under the $\mathrm{R} 6$ condition where maximal disruption should have resulted from the shift from six successive pairing trials to the first set of three test trials.

As for the minimal deleterious effects of increasing number of stimulus elements, this is consistent both with Newman's (1963) finding of a superiority for oneelement over multi-element S-terms on early trials which is eliminated or reversed on later trials, and Underwood \& Erlebacher's (1965) finding of probably insignificant decrements from increasing number of letter orders for dissimilar five-element $\mathrm{S}$-terms. Presenting multi-element s-terms in varying letter orders may well increase the likelihood of finding an easy association with the R-term, and thus counteract any deleterious effects of such letter-order variation. Postexperimental questioning revealed that under S3 conditions only one $S$ reported using all three letters for all eight pairs, and there was $56.8 \%$ usage of fewer than three letters combined over Ss and pairs. Thus letterorder variation does not eliminate stimulus selection. Pretraining, however, produced significantly $(p<.001)$ less stimulus selection (27.3\%), and six of the $20 \mathrm{Ss}$ reported using all three letters for all pairs. In any event, while the ineffectiveness of the present stimuluselement manipulation precludes any definitive conclusions, the absence of any postexperimental S-term recall differences between $R 1, R 3$, and $R 6$ conditions argue against stimulus learning as a major consequence of interpolated successive test trials.

\section{References}

Anderson, N. S. Word associations to individual letters. J. verbal Learn. verbal Behav., 1965, 4, 541-545.

Battig, W. F. Procedural problems in paired-associate learning research. Psychon. Monogr. Suppl., 1965, No. 1.

Lazar, G., \& Van Laer, J. Successive recall as a warm-tp task for paired adjectives. Psychon. Sci., 1966, 5, 137-138.

Newman, S. E. Performance during paired-associate training as a function of number of elements comprising the stimulus term. Tech. Rep. No. 4, Contract Nonr 486(08), 1963.

Reynolds, J. H. Paired-associate learning by anticipation and nonanticipation methods: Supplementary data. Psychol. Rep., 1965, $16,1123-1124$

Richardson, J., \& Gropper, M. S. Learning during recall trials. Psychol. Rep., 1964, 15, 551-560.

Underwood, B. J. Experimental psychology (2nd Ed.). New York: Appleton-Century-Crofts, 1966.

Underwood, B. J., \& Erlebacher, A. H. Studies of coding in verbal learning. Psychol. Monogr., 1965, 79, No. 13(Whole No. 606).

Underwood, B. J., \& Schulz, R. W. Meaningfulness and verbal learning. Philadelphia: Lippincott, 1960.

\section{Note}

1. This research was supported by Public Health Service Research Grant HD-01062 from the National Institute of Child Health and Human Development, and is based upon a Master's thesis by the senior author. 